\title{
PULMONARY ATELECTASIS AND OTHER RESPIRATORY COMPLICATIONS AFTER CARDIOPULMONARY BYPASS AND INVESTIGATION OF AETIOLOGICAL FACTORS
}

\author{
G.D. Gale, S.J. Teasdale, D.E. Sanders, * P.J. Bradwell, A. Russell, B. Solaric, \\ AND J.E. YORK
}

ATELECTASIS is a common respiratory complication after open heart operations with an incidence of 60 to 84 per cent (Table I). It is detrimental to patient recovery because it reduces lung compliance and increases the work of breathing and the alveolar-arterial oxygen gradient. This leads to hypoxaemia if the arterial oxygen tension cannot be maintained by an increase in the inspired oxygen concentration.

This study was designed to survey respiratory complications after heart operations with cardiopulmonary bypass and to investigate possible

TABLE I

\begin{tabular}{|c|c|c|}
\hline & Year & $\begin{array}{l}\text { Per cent incidence } \\
\text { of pulmonary } \\
\text { complications }\end{array}$ \\
\hline PROVAN, et al. ${ }^{1}$ & 1966 & 61.5 \\
\hline TEMPLETON, et $a l^{2}$ & 1966 & 74 \\
\hline GAUERT, et al. ${ }^{3}$ & 1971 & 70 \\
\hline TURNBULL, et $\mathrm{al}^{4}$ & 1974 & 84.3 \\
\hline GALE and SANDERS 5 & 1977 & 84 \\
\hline
\end{tabular}

From the Departments of Anaesthesia and Radiology, ${ }^{*}$ Toronto General Hospital, and University of Toronto, Toronto, Ontario, Canada.

Reprints from Dr. G.D. Gale, Department of Anaesthesia, 2nd Floor Norman Urquhart Wing, Toronto General Hospital, 101 College Street, Toronto, Ontario, Canada, M5G 1L7. aetiological factors. This was considered necessary to determine the effect of changes of technique, the haemodilution prime and the intraaortic balloon on a continued high rate of atelectasis which was unresponsive to treatment with the incentive spirometer when used four times a day. 5

\section{METHOD}

Clinical data were collected prospectively in 50 consecutive patients following heart operations with cardiopulmonary bypass. The mean age was 48.6 years (range $21-71$ ) and weight was $68.1 \mathrm{~kg}$ (range 40-94) with 34 males and 16 females. Two patients had radiological signs of chronic obstructive lung disease. Operations are shown in Table II. The intra-aortic balloon was used in one case after resection of left ventricular aneurysm and in eleven cases with aortocoronary bypass. The high rate of use of the balloon is accounted for by the fact that its indications had not been defined and it was being used prophylactically for stenosis of the left main coronary artery and poor left ventricular function. The mean stay in intensive care was four days.

Clinical examination of the chest has been found to under-estimate the frequency of atelectasis $^{5}$ and changes of temperature, pulse and res-

TABLE II

\begin{tabular}{lcc}
\hline \hline$\quad$ Operations performed in this study & $\begin{array}{c}\text { Number of } \\
\text { patients }\end{array}$ & $\begin{array}{c}\text { Patients with any } \\
\text { type of atelectasis }\end{array}$ \\
\hline $\begin{array}{l}\text { Mitral valve replacement } \\
\quad \text { One with double aorto-coronary bypass) }\end{array}$ & 4 & 2 \\
$\begin{array}{l}\text { Aortic valve replacement } \\
\quad \text { (one with ventriculomyotomy) }\end{array}$ & 8 & 5 \\
$\begin{array}{l}\text { Multiple valves (7 valves) } \\
\text { Aorto-coronary bypass } \\
\quad(59 \text { grafts and 11 balloons) }\end{array}$ & 3 & 2 \\
$\begin{array}{l}\text { Septal defects (3 ventricular, 3 atrial) } \\
\text { Resection of left ventricular aneurysm } \\
\quad \text { (with balloon) }\end{array}$ & 27 & 17 \\
$\begin{array}{l}\text { Ventriculomyotomy } \\
\text { Repair of descending thoracic aorta (redo) }\end{array}$ & 5 & 3 \\
Intra-aortic balloon & 1 & 1 \\
\hline
\end{tabular}


TABLE III

Radiological Survey Showing Number of Cases With Pulmonary Complications in Fifty Patients

\begin{tabular}{lr}
\hline \hline Effusions: Right (3 small 1 medium) & 4 \\
$\quad$ Left (12 small, 1 large) & 13 \\
$\quad$ Bilateral (13 small, 3 medium, 1 large, 2 mixed) & 19 \\
Total Effusions & 36 \\
Atelectasis & 32 \\
Paratracheal haematomata & 11 \\
Gastric dilatation & 7 \\
Signs of congestive heart failure & 5 \\
Tension pneumothorax (with chronic obstructive lung disease) & 1 \\
Left haemothorax, bilateral pneumonia, consolidation R.U.L. & 1 \\
Right basal consolidation & 1 \\
\hline
\end{tabular}

piration are poorly correlated with atelectasis after cardiopulmonary bypass, ${ }^{5}$ so respiratory complications were assessed radiologically. Preoperative chest films were reviewed. Films were then taken one to three hours after operation, at 24 and 48 hours and every two days thereafter unless required more frequently. A short time-exposure of one-twentieth of a second or less was used with a high kilovoltage technique to ensure good films. After extubation of the trachea films were taken in the upright position with additional lateral views.

Atelectasis was classified on radiological evidence as plate, subsegmental, segmental, or lobar in type. Plate atelectasis (synonymous with disk atelectasis) is described by Fraser and Paré ${ }^{6}$ and was originally described by Fleischner. ${ }^{7}$ It consists of linear densities almost always at the lung bases, roughly horizontal or slightly oblique, varying in thickness from one to three millimeters and commonly several centimeters in length. Lines due to plate atelectasis invariably extend to the pleural surface.

Statistical analysis was done using the chisquared test or Fisher's exact test of probability.

\section{RESULTS}

\section{Pulmonary complications: (Table III)}

The commonest finding was pleural effusion with an incidence of 72 per cent. Most effusions were small and there were more on the left side than the right. Effusions were unrelated to the distribution of atelectasis and were more frequent in patients with a cardiac index of less than two and one half litres per minute $(\mathrm{p}<0.02)$.

Atelectasis was the second commonest finding and is considered below. Paratracheal haematoma and gastric dilatation occurred in eleven and seven patients respectively. There were signs of congestive heart failure post-operatively in five patients including one with pulmonary oedema.

\section{Respiratory support and clinical progress}

Seven patients were ventilated for three to six hours after operation. Thirty-nine patients were ventilated overnight (from 12 to 24 hours). The remaining four patients required respiratory support for 37,52 and 65 hours and 14 days respectively. Thirty-two patients showed signs of reduced chest expansion, rhonchi or râles at the bases. Only two patients were unable to maintain an arterial oxygen tension of $100 \mathrm{~mm} \mathrm{Hg}$ $(13.3 \mathrm{kPa})$ when the inspired oxygen proportion was increased.

\section{Atelectasis}

\section{Incidence}

The overall incidence of atelectasis was 64 per cent (Table IV). Plate and subsegmental atelectasis was twice as common as segmental. There was only one patient with lobar atelectasis (incomplete right middle and lower) which was combined with a single left segmental atelectasis. Five other patients showed two types of atelectasis. There were three with plate and subsegmental and one each with plate and segmental

TABLE IV

The Incidence of Atelectasis in Fifty Consecutive PATIENTS

\begin{tabular}{lcc}
\hline & Number of patients & Per cent \\
\hline Any type & 32 & 64 \\
Plate & 15 & 30 \\
Subsegmental & 15 & 30 \\
Segmental & 7 & 14 \\
Lobar & 1 & 2 \\
\hline
\end{tabular}


TABLE $V$

Number of Patients With atelectasis Classified By Zone, OUt Of A TOtal OF Fifty Patients

\begin{tabular}{lccc}
\hline & Basal & Mid & Apical \\
\hline Any Type & 27 & 8 & 1 \\
Plate & 12 & 3 & 1 \\
Subsegmental & 11 & 6 & 0 \\
Segmental & 7 & 1 & 0 \\
Lobar & 1 & 1 & 0 \\
\hline
\end{tabular}

TABLE VI

The Number of Patients With Atelectasis Cl.assified By Side Out of a Total of Fifty Patients: the Three Categories Are Mutually Exclusive

\begin{tabular}{lccc}
\hline \hline & Right only & Left only & Bilateral \\
\hline Any Type & 11 & 7 & 14 \\
Plate & 4 & 4 & 7 \\
Subsegmental & 6 & 4 & 5 \\
Segmental & 2 & 3 & 2 \\
Lobar & 1 & 0 & 0 \\
\hline
\end{tabular}

and one with subsegmental and segmental atelectasis.

\section{Zone}

Analysis by zone showed basal atelectasis to be roughly three times as common as mid zone atelectasis (Table V). Apical atelectasis was rare and was present in only one patient who had a single left apical plate lesion combined with single bilateral basal plate atelectasis, a moderate right pleural effusion and a paratracheal haematoma.

\section{Side}

The frequency of unilateral atelectasis was similar on each side. Bilateral atelectasis occurred in 28 per cent of patients (Table VI).

\section{Aetiological factors}

\section{(1) Preoperative}

Analysis of data on age, obesity, preoperative pulmonary disease. productive cough, smoking habits, treatment with diuretics, pulmonary hypertension, poor left ventricular function (Grade III and IV) and raised left ventricular end-diastolic pressure above $12 \mathrm{~mm} \mathrm{Hg}(1.6 \mathrm{kPa})$ failed to correlate these factors with the occurrence of atelectasis.

\section{(2) Peroperative factors}

The use of positive end-expiratory pressure during operation, the degree of haemodilution as
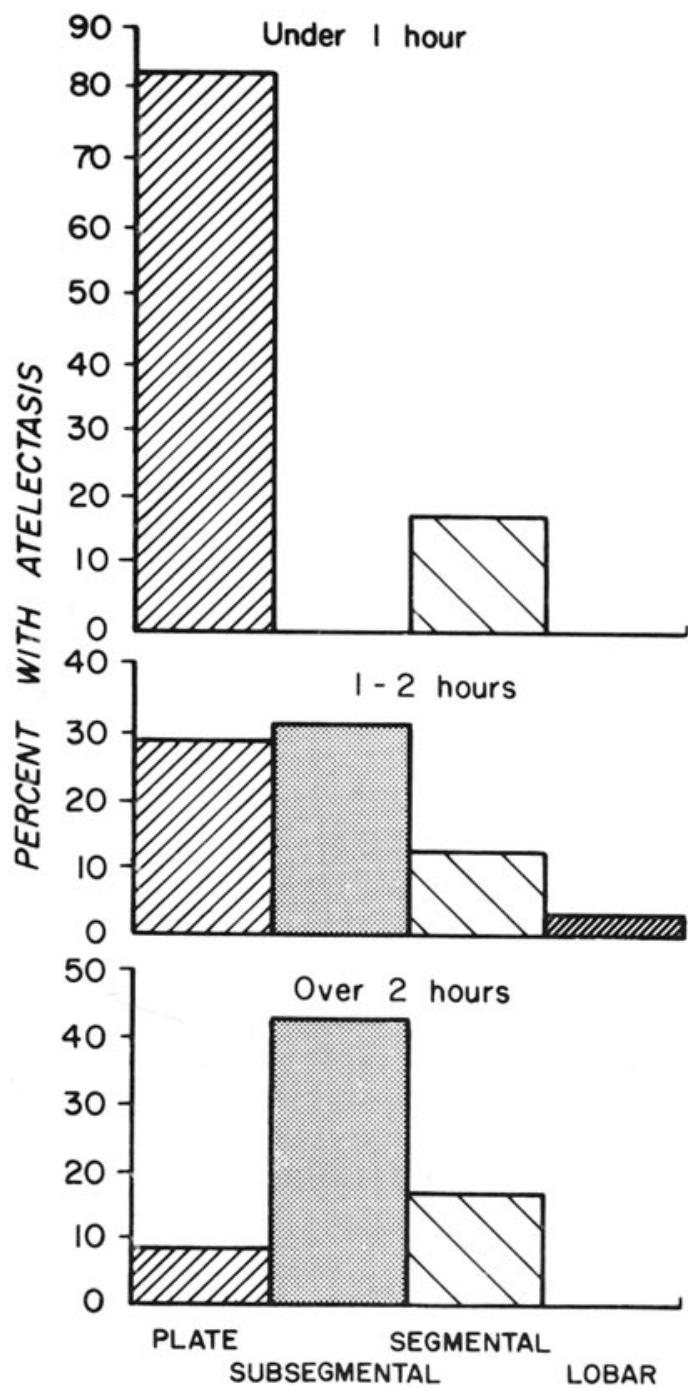

FIgUre 1 This shows the type of atelectasis found postoperatively after cardiopulmonary bypass times of under one hour, one to two hours and over three hours. The number of patients in the three groups was respectively 6, 32 and 12. Plate atelectasis was commoner in the group under one hour than in the other two groups $(\mathrm{p}<0.05$ and $<0.02)$.

judged by the last haematocrit on cardiopulmonary bypass and the use of the intra-aortic balloon were unrelated to the incidence of atelectasis.

Patients with a cardiopulmonary bypass time of less than one hour had a higher incidence of plate atelectasis than intermediate and long bypass groups ( $p<0.05$ and $<0.02$ ). In the longer bypass groups the incidence of subsegmental atelectasis rose but failed to reach significance (Figure 1). Segmental atelectasis was significantly more frequent if the positive fluid 

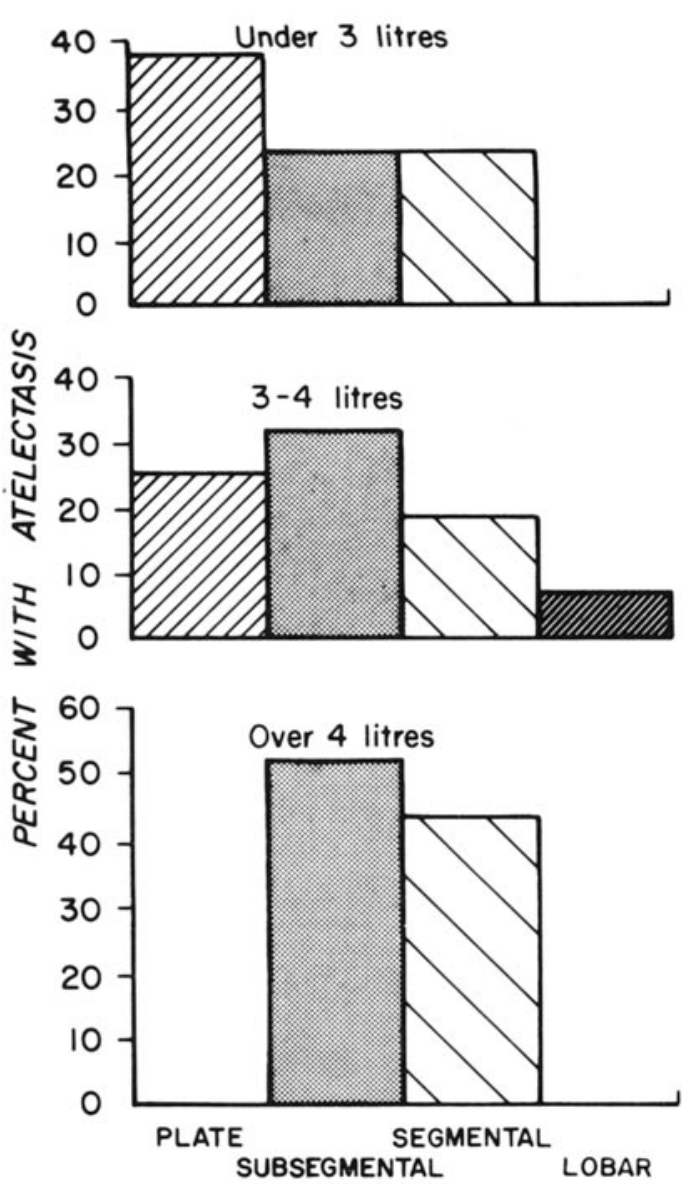

Figure 2 This shows the type of atelectasis found postoperatively in relation to the fluid load at the end of bypass, which is classified as under three litres, three to four litres and over four litres. The number of patients in each of the four groups was 26, 16 and 7 respectively. Segmental atelectasis was commoner in the group over four litres compared to those under three litres ( $\mathrm{p}<$ $0.05)$.

balance at the end of operation exceeded four litres compared to those in which it was less than three litres $(p<0.05)$ (Figure 2). There was a trend to less plate atelectasis and more subsegmental atelectasis in the group with a large fluid load but this failed to reach significance. Subsegmental atelectasis was significantly more common in patients who returned to the operating room for bleeding ( $p<0.05$ ) (Figure 3 ). Three patients in this group had thoracotomies, and another patient had a femoral balloon site explored for haematoma.

\section{(3) Postoperative factors}

Bleeding and atelectasis. Atelectasis occurred in nine out of eleven patients who lost more than one litre of blood in the first 24 hours after operation.

There was one case of right basal consolidation and nine cases of atelectasis out of eleven patients who had radiological evidence of paratracheal haematomata, but only four of these patients had over one litre of external blood loss. Only two patients with paratracheal haematomata were re-opened for bleeding.

Atelectasis occurred in all seven patients with gastric dilatation, which was usually found on the morning after operation $(p<0.05)$. Of seven patients with congestive heart failure four had atelectasis and one developed a right basal consolidation secondary to pulmonary oedema.

There was no correlation between the duration of postoperative ventilation and the incidence of atelectasis.

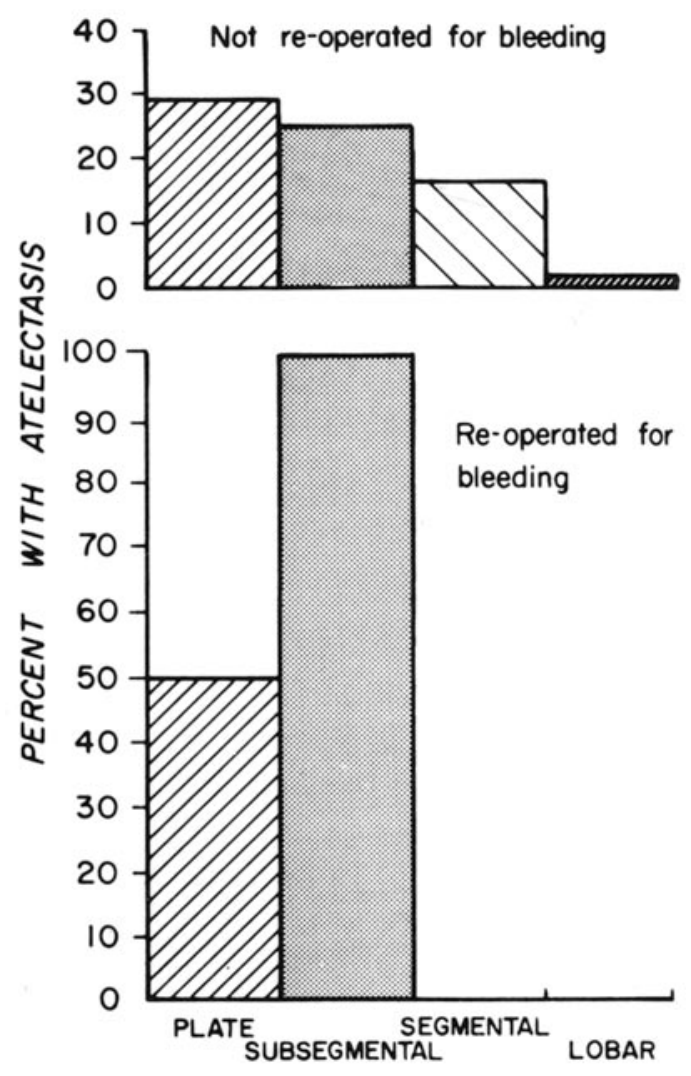

FIGURE 3 This shows the type of atelectasis in relation to re-operation for bleeding. Four patients out of 50 had re-operation for bleeding. Three had thoracotomies and one had exploration of a femoral balloon site. Subsegmental atelectasis was commoner in the re-operated group $(p<0.05)$. 


\section{Discussion}

Earlier papers agree roughly on the incidence of atelectasis after cardiopulmonary bypass but they differ on the distribution of atelectasis and aetiological factors.

\section{Incidence}

A frequency of atelectasis of 64 per cent is consistent with earlier observations which showed atelectasis occurring in 60 to 84 per cent of patients after cardiopulmonary bypass. ${ }^{1-5}$ The variation in incidence of atelectasis reported may be in part due to inconsistencies in frequency of radiological examination. The importance of a lateral chest X-ray is illustrated in one of our patients who showed atelectasis in one lateral view only. There may also be inconsistencies in the classification of atelectasis that is used. Provan' excluded minor radiological changes and this may account for his lower incidence of atelectasis.

\section{Distribution}

The results of this study agree with others that found more atelectasis at the bases than in the upper zones. ${ }^{2-5}$ Three earlier studies found more atelectasis at the left base. ${ }^{2-4}$ Our finding of the same incidence on each side agrees with an earlier study ${ }^{5}$ and suggests that in our patients there were no important left unilateral aetiological factors, such as compression of the lung from a large heart or surgical manipulation, unintentional intubation of the right main bronchus or failure to remove secretions from the left lung.

\section{Aetiological factors}

This study found that age, obesity and smoking habits were unrelated to the frequency of atelectasis. This might be explained partially by the fact that our patients were neither very old (only one was over 65 years) nor very obese. Gauert ${ }^{3}$ found that atelectasis increased with age after mitral but not after aortic valve replacements.

Our findings support the view of Hilberman ${ }^{8}$ that cardiac catheter data are a poor predictor of pulmonary function. The poor correlation between preoperative cardiac catheter data and postoperative atelectasis might be expected, since surgery is designed to improve cardiac function. The poor correlation between cardiac function and atelectasis continued into the recovery period, with atelectasis in only four of seven patients with congestive heart failure postoperatively.

The frequency of atelectasis was unrelated to the type of operation and the use of the intraaortic balloon. The former finding agrees with that of Gauert ${ }^{3}$ but not with that of Provan. ${ }^{1}$

More segmental atelectasis was found when the residual fluid balance after bypass exceeded four litres compared to those with less than three litres. This suggests that the increased lung water found after bypass ${ }^{9}$ causes atelectasis.

\section{Bypass time and atelectasis}

Our study found that the overall frequency of atelectasis was not increased in cases with a long bypass time in contrast to the findings of others. ${ }^{1,3.4 .10}$ More plate atelectasis was found in patients after a bypass time of less than one hour compared to those with a longer bypass. This finding is best explained by suggesting that many cases developed plate atelectasis within the first hour on bypass, but in longer bypass groups. atelectasis progressed from plate to subsegmental types (Figure 1).

In the short bypass group post-bypass fluid load and blood loss were low, so that the main causes of atelectasis were reduced vital capacity and residual volume and supine immobilization leading to small airway closure and basal atelectasis.

In the longer bypass groups additional factors after bypass were a larger fluid load. more bleeding and thoracotomies in three patients, which may have converted developing plate atelectasis into the more extensive subsegmental and segmental types. These three factors illustrate possible mechanisms of development of atelectasis. An increased water load after bypass will cause a rise in extra-vascular lung water which will reduce compliance, lung volume and ventilation, causing airway closure. Bleeding may cause atelectasis by pressure on the lung and atelectasis was present in nine out of eleven patients with paratracheal haematoma. Patients who have thoracotomy for bleeding are likely to get atelectasis from lung compression and trauma.

The mean duration of postoperative ventilation was the same in both short and long bypass groups, so that retention of bronchial secretions is a factor that would apply equally to both groups.

\section{Hypoxaemia and atelectasis}

After discontinuing controlled ventilation only 
two patients out of fifty were unable to maintain an arterial oxygen tension of $100 \mathrm{~mm} \mathrm{Hg}(13.3$ $\mathrm{kPa}$ ) with the use of an increased fraction of inspired oxygen. This suggests that although atelectasis was common, hypoxaemia was prevented in most patients. Atelectasis will matter most clinically where there is a large alveolararterial oxygen gradient. This may occur due to extensive atelectasis or due to interstitial pulmonary oedema occurring in heart failure or fluid overload. In these conditions arterial oxygenation cannot be maintained, lung compliance falls, the work of breathing is increased and the patient goes into cardio-respiratory failure. Our results did not show a correlation between atelectasis and postoperative heart failure but when they co-exist the problem of hypoxaemia is increased.

\section{Conclusions and Recommendations}

Although pleural effusion was the most frequent complication, most of these effusions were small and of little clinical significance and were unrelated to the incidence of atelectasis.

Atelectasis occurred in 64 per cent of patients. Investigation of possible aetiological factors was disappointing in not indicating more clearly a relationship of these factors to atelectasis.

Results suggest it is advisable to:

1. Restrict fluid load at the end of cardiopulmonary bypass.

2. Ensure adequate haemostasis and blood clotting after bypass to prevent blood loss, paratracheal haematomata and reoperation.

3. Prevent gastric dilatation by the use of a naso-gastric tube during and after operation.

4. Keep the time of cardiopulmonary bypass to a minimum.

5. Prevent and treat heart failure in the early postoperative period.

\section{SUMMARY}

Radiological evidence of pulmonary complications and possible aetiological factors were investigated in 50 consecutive patients after heart operations with cardiopulmonary bypass.

Atelectasis was the most frequent pulmonary complication except for small pleural effusions, with an incidence of 64 per cent. Several types of atelectasis frequently co-existed, with a predominance of the less extensive plate and sub- segmental forms. The incidence of atelectasis was the same on each side and the site of atelectasis was basal in three quarters of the patients.

Preoperative clinical and catheter data were unrelated to the incidence of atelectasis.

There was a significant positive correlation between a short cardiopulmonary bypass time and plate atelectasis, between a large fluid load after bypass and segmental atelectasis, between re-operation for bleeding and subsegmental atelectasis and between post-operative gastric dilation and atelectasis.

The type of operation. the use of the intraaortic balloon and the length of postoperative respiratory ventilation were unrelated to the incidence of atelectasis.

The mechanism of development of atelectasis is discussed.

\section{RÉSUMÉ}

Les manifestations radiologiques des complications respiratoires après chirurgie à coeur ouvert, ainsi que les facteurs étiologiques possibles de ces complications, ont fait l'objet d'une étude chez 50 malades consécutifs.

L'atélectasie s'est avérée la complication la plus fréquente (après les petites effusions pleurales) avec une incidence de 64 pour cent. Plusieurs types d'atélectasie ont fréquemment été observés en même temps chez un même individu. Les formes les moins importantes (atélectasie discoïde et atélectasie sous-segmentaire) ont été rencontrées les plus souvent. L'atélectasie se retrouvait le plus souvent aux bases $(3 / 4$ des cas) et elle se situait aussi fréquemment à droite qu“à gauche. On retrouvait plus souvent la forme discoïde d'atélectasie chez les malades ayant eu des circulations extracorporelles de courte durée; il y avait corrélation significative entre la forme segmentaire et une balance liquidienne positive importante en fin de C.E.C.. entre latélectasie de type sous-segmentaire et les interventions pour contrôle d'hémostase; il y avait de même corrélation significative entre l'incidence d'atélectasie et la présence de dilatation gastrique.

Par ailleurs, l'on n'a pu établir de corrélation entre lincidence d'atélectasie et les données cliniques et hémodynamiques pré-opératoires, la durée de C.E.C. ou l'emploi de contrepulsation intra-aortique.

Le travail discute du mécanisme de production de l'atélectasie. 


\section{ACKNOWLEDGEMENTS}

We are most grateful for support and encouragement in writing this paper from Professor A.A. Scott, for statistical analysis by Ms. B. Kuzin. and for financial assistance from the Marion Webster Taylor Fund of Toronto General Hospital.

\section{REFERENCES}

1. Provan, J.L., Austen, W.G., \& Scannell, J.G. Respiratory complications after open-heart surgery. J. Thoracic and Cardiovasc. Surg. 51:626 (1966).

2. Templeton, A.W., Almond, C.H., Seaber, A., Simmons, C.. \& MacKenzie, J. Postoperative pulmonary patterns following cardiopulmonary bypass. Am. J. Cardiol. 961: 1007 (1966).

3. Gauert, W.B., Anderson, D.S., Reed, W.A..\& Templeton, A.W. Pulmonary complications following extracorporeal circulation. Southern Med. J. $64: 679(1971)$.

4. Turnbull, K.W., Miyagishima, R.T., \& GeREIN, A.N. Pulmonary complications and car- diopulmonary bypass. A clinical study in adults. Canad. Anaesth. Soc. J. 21: 181 (1974).

5. Gale, G.D. \& Sanders, D.E. The BartlettEdwards incentive spirometer; a preliminary assessment of its use in the prevention of atelectasis after cardio-pulmonary bypass. Canad. Anaesth. Soc. J. 24: 408 (1977).

6. Fraser. R.G. \& Paré, J.A. Diagnosis of diseases of the chest. Volume 1,301. W.B. Saunders Company, Toronto (1970).

7. Fleischner, F. Uber das Wesen der basalan horizontalen shattenstreifen in Lungenfield. Wien, Arch. f inn. Med. 28: 461 (1936).

8. Hilberman, M., Kamm, B., Lamy, M., DietRICH, H.P., MARTZ, K., \& OSBORN, J.J. An analysis of potential physiological predictors of respiratory adequacy following cardiac surgery. J. Thorac. Cardiovasc. Surg. 7l: 711 (1976).

9. BYrick, R.J.. KaY, J.C.. \& Noble, W.H. Extravascular lung water accumulation in patients following coronary artery surgery. Canad. Anaesth. Soc. J. 24:332 (1977).

10. Anderson, N.B. \& ChIA, J. Pulmonary function, cardiac status, and postoperative course in relation to cardiopulmonary bypass. J. Thorac. Cardiovasc. Surg. 59: 474 (1970). 